\title{
ASSESSMENT OF PERFORMANCE CAPACITY AND POTENTIAL +*
}

\author{
J. D. BROOKE, D.L.C., M.Sc., Ph.D. \\ Physical Education Unit, University of Salford
}

The substance of this paper was presented to the conference 'Fitness for Sport' organised by the Sports Council,
Bisham Abbey, February, 1973.

One of the major concerns of sports scientists is the assessment of maximum performance ability and the prediction of maximum performance potential. The intention in the present paper is to examine these objectives, the level of success presently being achieved and the underlying concepts that today describe knowledge of such human performance. The topic examines the degree of commonalty at present existing at this part of the sports - science interface.

Let us first describe the manner of use of two key phrases. Maximum performance capacity is the highest level of a particular performance that a particular human being can achieve at the time of measurement. Maximum performance potential is the component to be added to the maximum performance capacity in order to establish the highest level of a particular performance that a human being will be able to achieve in the future. Capacity is the present ability. Potential is the additional latent behaviour that may be brought into use. Every coach and sportsman for whom work has been done in the Human Performance Laboratory at Salford has wanted to know (a) what is this sportsman's capacity and (b) what is his potential.

At this point a delimitation is introduced into the argument. The observations in this paper are made with the intention of considering the assessment of the top quality sports performer. This is the human being who might be classed in the top 100 in Great Britain for their type of sport, e.g. middle distance running or soccer playing. They are interested frequently in their performance within a small group of performers, perhaps around ten in number. Figure 1 illustrates this population, its derivation and typical sub-groups. So the problem is that of differentiating performance capacity or potential between such performers in these high quality homogeneous sub-groups of small numbers. Please note the object is differentiating between performers of high quality in these small homogeneous groups.

First, to Capacity. This is simple. To know to what level 'IT' can be done now, in the present setting, go and do it. Observing adequately it, the performance, the information is to hand immediately. If an athlete wants to know how fast he can run 100 metres, let him go, run and time it. Then repeat this, with suitable recovery allowed. Then perhaps repeat it again. The measure used

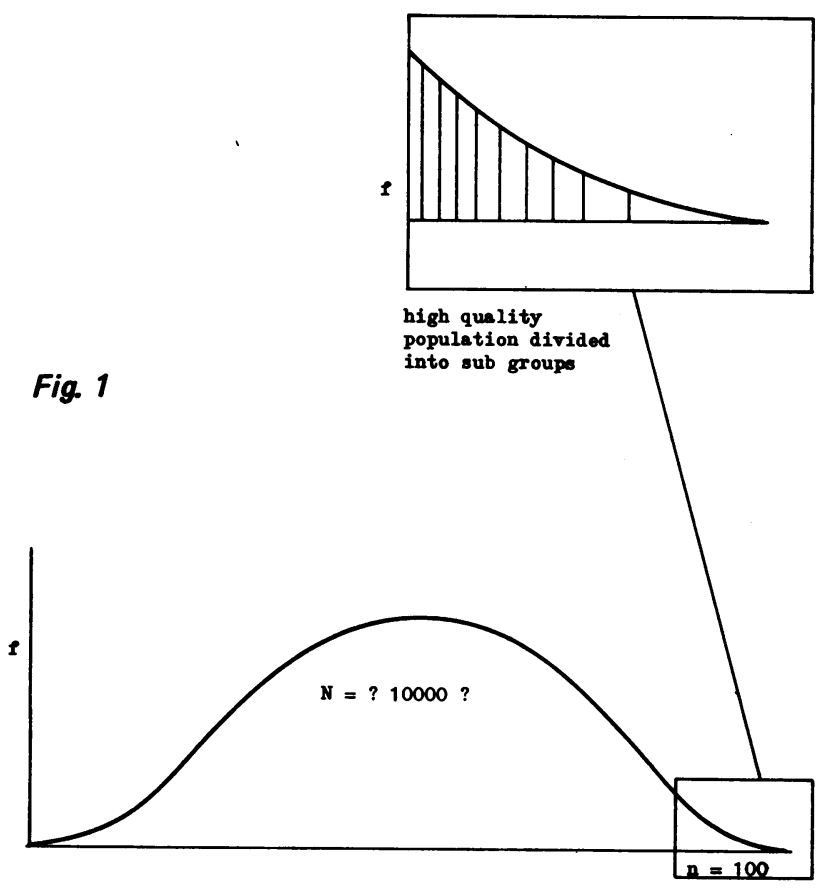

Population of active perticipants in the particular sporting activity

DIAGRAM OP HYPOTHETICAL HIGH QUALITI POPULATION, EXTRACTED FROM A MUCH LARGER POPULATION OP ACTIVE PARTICIPANTS IN THE PARTICULAR SPORTING ACTIVITI AND DIVIDED INTO TIPICAL SUB-GROUPS WITHIN WHICH INTER-PERPORMER COMPARISONS ARE REQUIRED

is valid logically. The variations between the times obtained will indicate the reliability, the degree to which the accuracy of measurement is less than perfect, that is the loss in replicability. There is no real problem in the assessment of such capacity.

Unfortunately in some cases this procedure is less than adequate. For example, it may be difficult to structure the environment in which the performance is to occur. Sufficiently sensitive timing devices (human or machine) may not be available, a large crowd of spectators may be an essential part of the scene, other performers, such as in team games, may be necessary, etc. Associated with such cases where assessment of capacity is required but the full performance conditions cannot be set up are also those cases where what is required is an assessment of potential, but with a very short time lag between the time of testing and of the real 
performance. Perhaps the true performance is to be the next day or the next week. In such cases the assumption is often that little basic change is anticipated in the preparedness to respond.

The obvious approach in both these cases is to measure the causative attributes that result in the performance. That is, if $P$ is the performance measure,

$$
P=a+b+c+d \ldots
$$

where $a, b, c, d$ are the causative attributes. So what are the human attributes that determine the particular characteristics of the performance?

For disease free subjects, one turns to the knowledge provided by the disciplines of Physiology, Psychology and Sociology in particular, and their interrelationships. This interrelated approach can be seen in the developing interest in Human Movement Studies as a field of study, e.g. Brooke and Whiting (1973).

As an example of attempting to identify the underlying variables determining performance, may we turn to the physiological aspects of the performance of activities such as middle-distance running. In doing this we select one of the areas of knowledge subjected to more detailed attention.

Let us overview some of this knowledge. As the physical power load required of the performer is increased, increased internal power is required also. This energy, generated externally by limbs moved by actin-myosin filament shifts contracting and relaxing muscle, is derived internally from two sources of supply:

(1) oxygen provision (the aerobic process) to permit re-synthesising of high energy bonds in muscle $(\mathrm{ATP}+\mathrm{PC})$ and

(2) the depletion of these high energy bonds with release of energy and incomplete re-synthesis (the anaerobic process).

Whenever possible $\mathrm{O}_{2}$ provision is used to maintain the high energy stores. As the external power demand on the performer increases (e.g. to run faster, that is increased $\mathrm{m} / \mathrm{kg} / \mathrm{s}$ or in the S.I. system increased W) proportional increases of response in $\mathrm{O}_{2}$ provision occur. Eventually with increasing demand a plateau occurs in provision and further increases in external power. are met only by use of the finite high energy stores, which depletion is associated with exhaustion. As shown in Fig. 2 the state of adequate $\mathrm{O}_{2}$ provision we term homeostasis (for that function) and when there is inadequacy of provision the state is termed disequilibrium. The oxygen is taken in at the lungs, carried in the blood pumped by the heart and off loaded at the tissues. With inadequate oxygen supply the lack of re-synthesis of high-energy products is seen in the release of lactic acid from muscle, which is observed

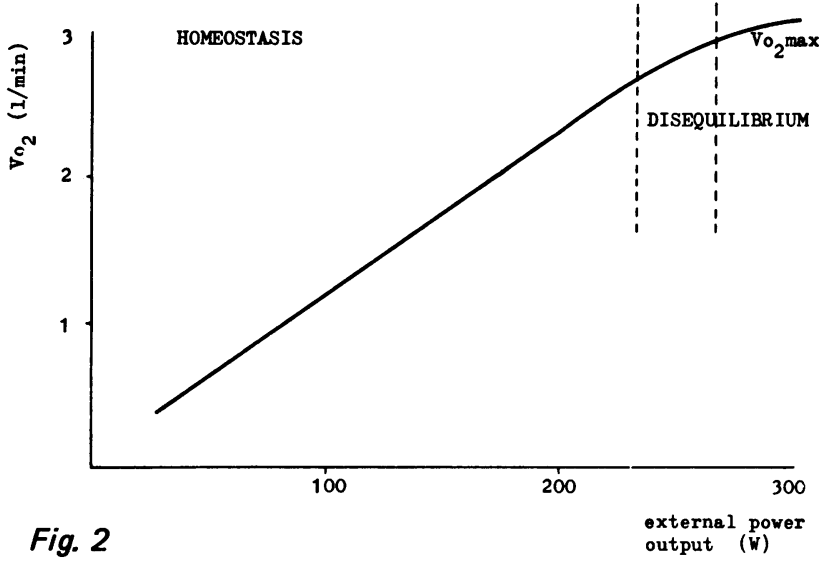

TIPICAL CURVE OF OXYGEN UPTAKE AGAINST EXTERNAL POWER OUTPUT INCREASING TO INCAPACITI

buffered in the blood as lactate. Alternatively the $\left(\mathrm{cH}^{+}\right)$ of the blood may be assayed. For work of ten to fifteen minutes in commonly encountered conditions this is an adequate survey of the system that will show limits with increasing work. But put together the data presently taken from this system, working from the principle of $P=a+b+c+d \ldots$ and it is proposed that the resultant variance found in these underlying variables has not yet been shown (in published material) to discriminate adequately across a range of work performance such as that seen in our homogeneous group of runners. Take $\mathrm{VO}_{2}, V_{E}, F_{1}-F_{E O}, F_{E V}, D_{L}, D_{M}, V_{C}, H f \max , H f$ quadratic curvature, $\mathrm{Hf}$ basal (with such people $\dot{Q}$ is inferred almost always from $\mathrm{Hf}$ ), ECG wave form, $\mathrm{PaO}_{2}$, arterial blood post exhaustion $\left(\mathrm{CH}^{+}\right)$highpoint (or lactate concentration) and relate them to a performance criterion such as maximum power output tolerated or speed of run. Avoiding statistical monsters (such as multiply regressing as many dependent variables as you have subjects' scores in the independent variable!) correlation between performance and underlying variables has not been high in such groups. Treatments of data for body weight or partial order correlating to remove age effects etc., do not much alter the result in a problem such as the present one. There are pieces of data yet to be obtained and these may help (e.g. level of respiratory enzyme activity to mitochondria or levels of various hormones released with exercise). The fact remains that very few studies can be found where differences in performances in such a group are well accounted for by differences in the underlying variables commonly identified. One of the more successful may be Ishiko (1967) showing in 8 Japanese Olympic trialist middle-distance runners a significant correlation of 0.67 between best 5000 metre run time and maximal oxygen uptake per $\mathrm{kg}$ body weight per minute. Note, however, the mean maximum $\mathrm{VO}_{2} / \mathrm{kg}$ of $45.3 \mathrm{ml} / \mathrm{kg} /$ minute for 
the group was low by British standards and the range shown of $\pm 6 \mathrm{ml}$ is wide (it is not clear whether this is \pm $S_{i}, \pm 1.96 S_{i}, \pm S_{\bar{x}}$, or $\left.\pm t_{s} \bar{x}\right)$. Searching through the literature and analysing carefully the few results that are at first discovered, the information is inadequate.

There are some matters to advance to account partly for this inadequacy. Firstly, the problem of Specificity. In the present discussion this is the problem of how close to the real life performance is the behaviour that it is proposed to measure as a performance criterion. It is very probable that the more apart the two behaviours are, the greater the differences to be expected in the functioning of the underlying attributes. If these attributes have functioned in a particular manner, because of repeated practice of a particular type of performance, then to assess their function with a different performance may, in homogeneous highly trained groups, generate so much additional variance that the original variance which critically differentiated one performer from another is swamped. When a performance criterion is to be used as substitute for the real performance the correct course of action is to make a best approximation of the former to the latter. For runners this indicates a treadmill and for racing cyclists the newly developed Ergowheel* ${ }^{*}$ where the habitual furniture of the cyclist's own bicycle frame is connected to a calibrated loading system. (For lay fitness assessment it probably does not matter). With telemetry of many human functions in its infancy we do not know how well such approximations replicate the true field demands: for many situations we do not know how valid the performance criterion is to the performance.

\section{*Worthwhile Designs, Grange Road, Eccles.}

Whilst considering specificity, what then of the use of dynamometers, jump boards and the like? May the repeated difficulty in relating such measures to many field situations of homogeneous high quality groups of human beings, lie in the high individual specificity of these measures and low commonalty with the field situation?

Secondly, under mitigating factors, we must ask how replicable are these variables - both performance criteria and physiological. All too often reliability coefficients are not quoted and when they are there is a wide range. In recent data on sportsmen of the type we are considering, working in our own laboratory on the treadmill to exhaustion with a mean work time of $\mathbf{2 7 0}$ seconds, we observed test-retest times for individuals which varied maximally by approximately 9 seconds (95\% confidence limits). With the narrow span of the group results this produced a zero order reliability coefficient! Now if we cannot replicate the performance criterion there is not much point in inferring either towards differences in the real performance or back to differences between individuals in the underlying variables. In the above instance the problem was alleviated somewhat by the use of the sum of repeated maximal testing which reduced still further individual variability so that a reliability coefficient of 0.82 resulted.

Again for the physiological variables, reliability coefficients do range greatly. They must be checked and stated if any interpretation is to be made of results. From our experience heart rate measures are the most reliable, but often not much related to maximum performance criteria without quite complex statistical analysis, cf Brooke \& Hamley (1972). Oxygen uptake measures also often are quite reliable $(0.8$ to 0.9$)$ and more related but moving to areas such as the $\left(\mathrm{cH}^{+}\right)$ highpoint of blood post-exhaustion, adequate replication can be very difficult to obtain.

It is worth noting two general points about reliability coefficients.

1. A reliability coefficient compares the variability in measuring each individual from measure to measure with the variation found between the subjects in the group. It is a comparative not an absolute statement.

2. If a reliability coefficient (using Pearson's product moment correlation) is 0.7 it means approximately $49 \%$ of the variance is common to two attempts to measure the same attribute and that approximately $51 \%$ of the measurement scores variance is not common. Such high specific variance markedly reduces the possibility of relating that attribute (measured in that way) with another attribute. From these two points may derive some of the problems in attempting to relate underlying physiological functions to differences in performances of the type under consideration.

This is the state of capacity evaluation from known causative variables in one of the more explored areas of sports science. What of the less well explored. Here attention is being directed to, e.g., the delicate skill performances involved in golf, snooker, fly-fishing, tennis, rugby, soccer, squash, netball, etc. The challenge is immense and exciting: with due professional respect (fully meant) results so far offer little to the solution of the present problem. Searching for psychological and sociological characteristics which permit differentiation within our homogeneous trained groups, there is little published information available to date. Much more work is required even in those areas where the most effort is occurring such as ball skill acquisition, or personality assessment by questionnaire or interview.

This is the state of study of such performance capacity. What then of the assessment of performance potential. It is felt that much of the lack of precision in 
assessing capacity carries over to attempts to assess potential. But so often it is just the potential about which information is required. Will the performer swim well next month? Has the performer the potential to win gold medals in three years time, etc., etc.?

However, more is involved in the assessment of potential for there is the implication of change, of adaptation. There is the suggestion that events will activate latent behaviour and make it patent. What do we know of adaptation? The cardio-respiratory physiology of the topic is reviewed by Brooke \& Knowles (1973) and it will suffice for the present to make some comments. Adaptation can occur either positively, resulting in hypertrophy from a physiological viewpoint, and this is the commonly observed training or practice effect, or it can occur negatively, with resultant hypotrophy, seen for example in middle age degeneration when sports activities are given up. There is biological adaptation when physico-chemical trophic changes take place. There is also psychological adaptation when readjustment of a psychological level or setting occurs. For more details on the latter, see e.g. Helson (1964) and his proposals on adaptation levels. In exercise physiology it is accepted that gains in muscle power result from progressively overloading the muscle and it is proposed by some that the more specific the training activity is to the real performance, the more likely it will be that adaptation in the maximum performance capacity will occur.

One very interesting aspect of adaptation recently to be uncovered is considered in Brooke \& Davies (1973) review of nutrition and endurance performance. Briefly, adaptation of the muscle glycogen stores to a higher level occurs following exercise to exhaust much of the initial store and a subsequent carbohydrate rich diet, c.f. Saltin \& Hermansen (1967). In such cases the performance capacity of our type of performer can double, dependent upon this quite short term adaptation, Brooke \& Green (1972). Its application is primarily to human movement vigorously sustained over hours, Brooke (1973).

But mainly these are fundamental starting points in defining the structure of knowledge about adaptation for sport. They do little to provide answers to some of the key problems. For example, what type of progressive overload training, that is specific by speed or by distance or without reference to specificity? How often should a performer train, per day, per week, per month? How long is it necessary to train? Is a particular performer over-trained? What are the principles of peaking? etc., etc. These are the questions that are asked and it is proposed that the scientific literature does not supply the answers. The science of sports adaptation (not folk account or dogma but a scientifically assembled structure from or of data) is fragmented even from the most optimistic viewpoint.
So, having painted this Bosch-like picture of the Garden of Earthly Delights where much is chaos, a destructive picture, what of the constructive? Let us make two statements summarising the above and then some observations.

1. Elastic changes across the range of capacity can be followed in some underlying variables but there is still missing a significant section, necessary to account for performance now.

2. Plastic changes in the range of adaptation - the range of latent potential - we follow little the relevant variables and there seems to be much missing.

It seems clear that what is required if there is to be adequate assessment of performance capacity and performance potential is for there to be intelligent inter-relationship of variables from the range of disciplines within human movement studies so that the key attributes may be identified. Probably these will not be weighted in the same way for each individual, for such rigidity does not often characterise human performance. It is more common to see within a human population variations in the functional manner with which objectives are attained. This flexibility of approach within the species is probably an important aspect of species survival. In successful scientific study of the characteristics of the performers in our trained? homogeneous groups, it will be necessary through multivariate analysis to sift out those variables that are not accounting for differences in performance ability and to pinpoint others, or combinations of others that account well for these differences. It may well be that variables will be discarded which are known to be vitally important to the effecting of the performance but which do not show at that level of performance variation linked to variations in the performance. Speculating, it seems worthwhile to turn to evidence of increased respiratory enzyme and metabolic (carbohydrate) enzyme activity at adapted muscle, to hormone release (both for longer term adaptation and for preparation to perform), to the whole area of arousal to perform and attention to task related and task unrelated information from the environment, social and inanimate, and in particular to neural activity associated with both arousal generally and also specifically with skeletal muscle innervation. I am sure that a researcher from a different standpoint could repeat that length of statement and suggest a different list of variables. This is a personal direction of thoughts. One must look to the attributes and look to the types of adaptation with training. To do this in a multivariate manner, the research must be large scale. Therefore, one must look to the money bags. Without research grant support the work cannot be done. To pay the cost of employing one technician for one year now is in the region of $£ 1,750$. The need is for 
a number of technicians and researchers with sufficient apparatus over a number of years. Without such efforts the answers may be a long time in arriving.

Let it be reiterated once more. This paper is concerned with the problem of assessment of performance and potential in small groups of highly trained and competent sportsmen. It cannot be generalised to work on the unfit, or to populations encompassing the highly competent down to the much less competent. It is the homogeneous nature of the groups that causes the problems identified. Unfortunately, it is such groups that interest most people coaching to achieve a high level of performance.

It seems that humility and endeavour without false claims appears to be the role of the sports scientist dealing with matters at present. Pseudo-science must be avoided. Claims to the glory of differentiating between performers of the type discussed or of predicting such differences in latent potential should be subjected to careful scrutiny. In such a context of limited ability the coach's 'empathy' with the performer and his knowledge which has accumulated over years almost without his awareness, remain very important in achieving high quality performance.

\section{Acknowledgement}

I am indebted to the contributions resulting from a number of discussions with Mr. J. E. Knowles, B.Sc., a research student in this Unit.

\section{REFERENCES}

1. BROOKE, J. D. Food carbohydrates and human performance. In: G. G. Birch \& L. F. Green (Eds.). Molecular Structure and Functions of Food Carbohydrates. London: Elsevier, 1973.

2. BROOKE, J. D. \& DAVIES. G. J. Nutrition and endurance performance - Abstract. Br.J.Spt.Med. (1973, 7, Nos. 3/4). 3. BROOKE, J. D. \& HAMLEY, E. J. The heart rate - physical work curve analysis for the prediction of exhausting
work ability. Med.Sci.Spt. 4, 1, 1972, 23-26.

4. BROOKE, J. D. \& KNOWLES, J. E. A review of Physiological adaptation to physical training for endurance. - Abstract. Br.J.Spt.Med. (1973), 7 Nos. 3/4.

5. BROOKE, J. D. \& WHITING, H. T. A. (Eds.). Human Movement - A Field of Study. London: Kimpton, 1973.

6. HELSON, H. Adaptation Level Theory. New York: Harper, 1964.

7. ISHIKO, T. Aerobic capacity and external criteria of performance. Can.Med.Ass.J. 96, 1967, 746-749. 1967, $746-749$. 